SCIENCE CHINA

Physics, Mechanics \& Astronomy

\title{
Application of THz technology in oil and gas optics
}

\author{
XinYang Miao, HongLei Zhan, and Kun Zhao* \\ Beijing Key Laboratory of Optical Detection Technology for Oil and Gas, China University of Petroleum, Beijing 102249, China
}

Received November 11, 2016; accepted November 24, 2016; published online December 20, 2016

$\begin{array}{ll}\text { Citation: } & \text { X. Y. Miao, H. L. Zhan, and K. Zhao, Application of THz technology in oil and gas optics, Sci. China-Phys. Mech. Astron. 60, 024231 (2017), doi: } \\ & \text { 10.1007/s11433-016-0452-9 }\end{array}$

Fossil fuels currently satisfy $\sim 88 \%$ of China's energy demands. Oil-gas, known as the hydrocarbon occurred from various types of underground strata, is the most significant part of primary energy sources. Problems in oil-gas exploration, transportation and petrochemical affect the economic viability of the petroleum industry. Recently, the increasing complexity of stratigraphic condition and the fall in oil prices has made the situation more critical. Thus, questing for new technologies is never ending. Optics, a general method of passing information to and from materials, can be served as an appropriate method to promote the development of oil-gas industry. Terahertz $(\mathrm{THz})$ radiation, which is located between far-IR (infra-red) and millimeter-wave bands of the spectrum, is willing to be a "silver bullet" in various aspects of oil-gas resources owing to the unique advantages. By using a THz system with appropriate scan ranges, the mixture of complicated components can be precisely characterized [1]. In this paper, some recent progresses of applied $\mathrm{THz}$ technique in various research areas of oil-gas optics detection are presented, including exploration and development of oil-gas reservoirs, transportation of oil-gas as well as evaluation of petrochemicals and pollutants (shown in Figure 1).

Characterization of oil-gas reservoirs underground includes the properties of organic matters and rocks. As a kind of hydrocarbon-generating parent material, kerogen is a complicated mixture of organic components, with the composition and pyrolysis process obscure to researchers. THz measurements have built a relationship between the absorption coefficient $(\alpha)$ and hydrocarbon evolutionary stage $\left(R_{0} \%\right)$. Si-

*Corresponding author (email: zhk@cup.edu.cn) multaneous characterization of the kerogen's oil- and gasgenerating peaks was achieved by plotting THz- $\alpha$ with $R_{0} \%$ (shown in Figure 1(b)), indicating great promise of THz technology to improve kerogen analysis [2]. Oil shale, defined as a finely grained sedimentary rock with kerogen contained, yields substantial amounts of oil and combustible gas by destructive distillation. As an important indicator to optimize the comprehensive utilization, oil content of the pyrolytic oil shale (semicoke) was observed to be associated with $\mathrm{THz}-\alpha$, indicating $\mathrm{THz}$ technology could be an effective selection for evaluating the oil content of oil shale [3]. In addition to the organic matters, characterizing the mineral's evolution of a reservoir is also essential to the development of the resource. Diagenetic stages (e.g. eogenetic stage, telogenetic stage and metamorphic stage) of halite rock were stated by $\mathrm{THz}-\alpha$ with the temperature [4], proving that $\mathrm{THz}$ wave was effective in geological research.

Better understanding of the reservoir's seepage law during the mining process is essential to improve oil recovery and predict future performance of the oil field, especially for the low permeability reservoirs. An argillaceous sandstone reservoir core with the permeability 1.0 millidarcy was measured with a reflective THz-TDS. Slices of the core were numbered according to their original positions and scanned by $\mathrm{THz}-$ TDS via mechanical motion to obtain the distribution of oil and water in the entire core. As shown in Figure 1(c), the existence of kerosene, water and remaining oil was clearly displayed by $\mathrm{THz}$ tomography, with the gradually varied color related to the changing of water content in the core [5].

After exploited from wells and pretreated preliminarily, crude oil and natural gas will be transported with pipelines 

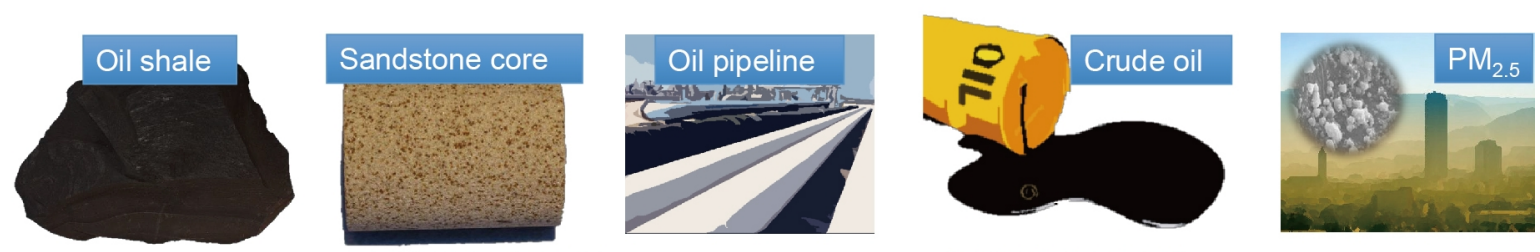

(a)
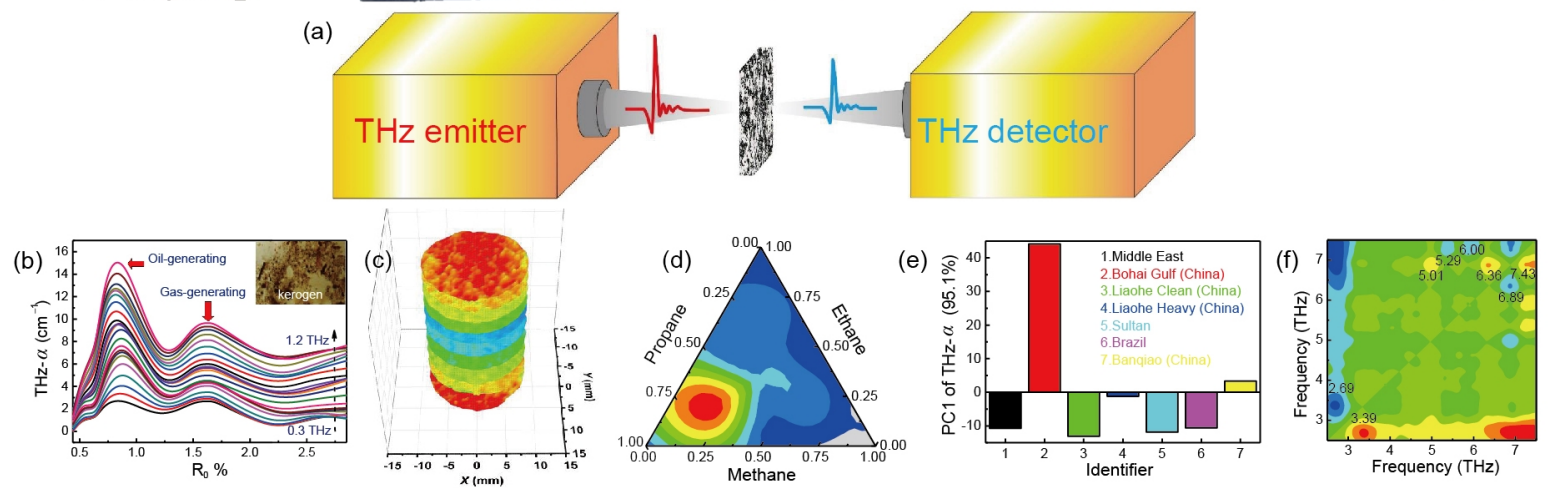

Figure 1 (Color online) (a) Schematic diagram of THz spectroscopy and various test results: (b) maturity dependent THz- $\alpha$ of the kerogen; (c) THz tomography characterized the oil and water distribution in a core; (d) phase projection picture of the $\mathrm{CH}_{4}+\mathrm{C}_{2} \mathrm{H}_{6}+\mathrm{C}_{3} \mathrm{H}_{8}$ system; (e) crude oil classification by the first principle component (PC1) of THz- $\alpha$; (f) 2-D correlation plot of $\mathrm{PM}_{2.5}$ over the frequency range 2.5-7.5 THz.

and other tools. Identifying the sources of crude oil and components of natural gas in the pipeline is of great importance for subsequent processing. Crude oils from different oil fields and principal components of natural gas were investigated with the combination of $\mathrm{THz}$ technique as well as multivariate statistical methods including cluster analysis and principle component analysis. The combination was able to classify the crude oils with different geographical locations [6] and quantify the hydrocarbon components of natural gas [7], which were exhibited in Figures 1(d) and (e). Besides, oil and water two-phase flow in horizontal pipes is a common occurrence in the petroleum industry for the transportation of crude oil. The investigation of pattern transitions is very important in horizontal oil-water two-phase flow system, especially for the measurement of flow parameters and optimization of industrial production process. THz-TDS was employed to characterize the water content and the pattern transitions in the oil-water two-phase flow simultaneously. The amplitude of the $\mathrm{THz}$ pulse was correlated to the water content, and the critical flow rates of the flow pattern transitions point show a non-linear trend with the water content. This work provided an in situ means to investigate the flow system in the process of crude oil transportation.

Additionally, non-destructive testing of the pipeline as well as the oil tank is a preparatory work to prevent oil leaks, efficient and safe methods are also required for gas detection, storage and transportation. A simulated sample with crude oil filled was illuminated with THz-TDS, and the oil-gas interface was clearly shown by $\mathrm{THz}$ images and spectra [8]. We also monitored the formation and decomposition processes of methane hydrates by $\mathrm{THz}$ amplitude changed over time. In our experiment, the cage-structure of hydrates formed gradu- ally with the decreasing temperature of $272.2-266.2 \mathrm{~K}$, and remained stable when the temperature goes up to $272.2 \mathrm{~K}$ owing to the Van der Waals forces between $\mathrm{CH}_{4}$ and $\mathrm{H}_{2} \mathrm{O}$ molecules. Accordingly, $\mathrm{THz}$ amplitude rose during the initial freezing stage, and maintained a nearly constant value with the decreasing pressure and rising temperature [9]. The results demonstrated the unique capability of THz-TDS to monitor the synthesis of gas hydrate, which were helpful to the gas storage and transportation researches.

As one of the most important sources of fuels and raw materials, petrochemicals have made remarkable contributions to the development of human civilization. Owing to the sensitivity of low-frequency vibration and rotation modes, $\mathrm{THz}$ technology is considered to be a reliable analytic method in liquid petrochemicals detection. Qualitative analyses of lubricant, gasoline and diesel were performed. Quantitative detection of their additives was also carried out by analysis with BP artificial neural networks (BPANN) and support vector machine. Typical concentrations of sulfur in gasoline (ppmgrade) could be detected, together with methyl methacrylate in diesel below $0.5 \%[10,11]$.

$\mathrm{PM}_{2.5}$, defined as the particulate matters in an atmosphere with diameters of no more than 2.5 microns, has attracted extensive attention recently owing to the health impacts to human beings. Therefore, techniques for monitoring $\mathrm{PM}_{2.5}$ are of great significance. In our studies, $\mathrm{THz}$ spectra of $\mathrm{PM}_{2.5}$ with various concentrations were measured by Fourier transform infrared spectrometer [12] and THz-TDS $[13,14]$, with larger signal to noise ratio in relatively high and low frequencies, respectively. Partial least squares and BPANN were employed to quantitatively characterize the $\mathrm{PM}_{2.5}$ content with the input of absorbance over 0.1-2 and 
2.5-7.5 THz, the elemental compositions were also studied by monitoring $\mathrm{PM}_{2.5}$ masses in conjunction with two-dimensional correlation spectroscopy (shown in Figures 1(f)). The results could be used by environmental policymakers and experts to develop appropriate plans and techniques for PM control.

In summary, $\mathrm{THz}$ spectroscopy has been proved to be a promising means for determining oil-gas resources and their products. Therefore, as an emerging technology, THz technique is willing to promote the development of oil and gas optics in the future.

This work was supported by the National Natural Science Foundation of China (Grant No. 11574401), the National Basic Research Program of China (Grant No. 2014CB744302), the Specially Founded Program on National Key Scientific Instruments and Equipment Development, China (Grant No. 2012YQ140005), and the China Petroleum and Chemical Industry Association Science and Technology Guidance Program (Grant No. 2016-01-07).

1 H. L. Zhan, S. N. Sun, K. Zhao, W. X. Leng, R. M. Bao, L. Z. Xiao, and Z. W. Zhang, Sci. China Technol. Sci. 58, 2104 (2015).

2 R. M. Bao, S. X. Wu, K. Zhao, L. J. Zheng, and C. H. Xu, Sci. ChinaPhys. Mech. Astron. 56, 1603 (2013).
3 R. M. Bao, Y. Z. Li, H. L. Zhan, K. Zhao, W. Wang, Y. Ma, J. X. Wu, S. H. Liu, S. Y. Li, and L. Z. Xiao, Sci. China-Phys. Mech. Astron. 58, 114211 (2015).

4 R. M. Bao, Q. Meng, C. L. Wang, T. Y. Cai, C. Dong, H. L. Zhan, X. Y. Miao, C. J. Feng, L. Y. Zhang, and L. Z. Xiao, Sci. Sin.-Phys. Mech. Astron. 45, 084203 (2015).

5 R. M. Bao, X. Y. Miao, C. J. Feng, Y. Z. Zhang, H. L. Zhan, K. Zhao, M. R. Wang, and J. Q. Yao, Sci. China-Phys. Mech. Astron. 59, 664201 (2016).

6 H. Zhan, S. Wu, R. Bao, L. Ge, and K. Zhao, Fuel 143, 189 (2015).

7 L. N. Ge, H. L. Zhan, W. X. Leng, K. Zhao, and L. Z. Xiao, Energy Fuels 29, 1622 (2015).

8 D. D. Wang, X. Y. Miao, H. L. Zhan, J. Wang, and K. Zhao, Sci. China-Phys. Mech. Astron. 59, 674221 (2016).

9 X. Y. Miao, S. N. Sun, Y. Z. Li, W. Wang, R. M. Bao, and K. Zhao, Sci. China-Phys. Mech. Astron. 60, 014221 (2017).

10 H. Zhan, K. Zhao, H. Zhao, Q. Li, S. Zhu, and L. Xiao, J. Phys. D-Appl. Phys. 49, 395101 (2016)

11 F. L. Qin, Q. Li, H. L. Zhan, W. J. Jin, H. L. Liu, and K. Zhao, Sci. China-Phys. Mech. Astron. 57, 1404 (2014).

12 H. Zhan, K. Zhao, R. Bao, and L. Xiao, J Infrared Milli Terahz Waves 37, 929 (2016).

13 H. Zhan, Q. Li, K. Zhao, L. Zhang, Z. Zhang, C. Zhang, and L. Xiao, IEEE Trans. THz Sci. Technol. 5, 1028 (2015).

14 H. L. Zhan, K. Zhao, and L. Z. Xiao, Sci. China-Phys. Mech. Astron. 59, 644201 (2016). 